\title{
Knowledge and Awareness with regard to Oral Cancer and Its Risk Factors among Medical and Dental Students at Majmaah University
}

\author{
Turki M. Bin Mahfoz ${ }^{1}$ \\ ${ }^{1}$ Department of Otolaryngology, College of Medicine, Al Imam \\ Mohammad Ibn Saud Islamic University (IMSIU), Riyadh, Saudi Arabia.
}

\section{ABSTRACT}

\section{BACKGROUND}

The incidence of human papillomavirus (HPV) related oral cancer is increasing globally. This study was carried out with an aim to assess the knowledge and awareness of oral cancer and its risk factors among medical and dental students in Saudi Arabia.

\section{METHODS}

This cross-sectional study was conducted in Majmaah University, Saudi Arabia, between April and May 2020. A questionnaire was distributed among 343 male and female dental and medical students, who were selected by simple random sampling. Questionnaire was distributed to determine their awareness and knowledge about oral cancer and its risk factors. Their responses were analysed using SPSS version 22 software.

\section{RESULTS}

Three hundred and forty-three completed questionnaires were available for analysis. Seventy percent of the respondents were males, $47 \%$ were dental students, and 53 $\%$ were medical students. Most (84 \%) of respondents agreed strongly that smoking is a risk factor for oral cancer; $70 \%$ agreed strongly that chewing powdered tobacco products like gutka was another risk factor. However, only $25 \%$ of students were aware that HPV could cause oral cancer. Overall, $53 \%$ of students had moderate knowledge, $12 \%$ had good knowledge, $29 \%$ had poor knowledge, and $6 \%$ had no knowledge that HPV was a risk factor. Gender had no effect on depth of knowledge. However, medical students were more knowledgeable about HPV related oral cancer than were dental students.

\section{CONCLUSIONS}

Dental and medical students at Majmaah University were not sufficiently informed about oral cancer or the relationship between HPV and oral cancer. Awareness courses and modification of the curriculum to include the risk factors for oral cancer, particularly the relationship between HPV and oral cancer, are recommended.

\section{KEY WORDS}

Oral Cancer, Risk Factors, Human Papillomavirus, Awareness, Medical Students, Dental Students, Saudi Arabia

\section{Corresponding Author: Dr. Turki M. Bin Mahfoz, Department of Otolaryngology, College of Medicine, Al Imam Mohammad Ibn Saud Islamic University, (IMSIU), Riyadh, Saudi Arabia E-mail: tmmahfoz@imamu.edu.sa}

DOI: $10.14260 /$ jemds/2021/102

How to Cite This Article:

Bin Mahfoz TM. Knowledge and awareness with regard to oral cancer and its risk factors among medical and dental students at Majmaah University. J Evolution Med Dent Sci 2021;10(08):463-468, DOI: $10.14260 /$ jemds/2021/102

Submission 27-08-2020,

Peer Review 24-12-2020,

Acceptance 30-12-2020,

Published 22-02-2021.

Copyright (C) 2021 Turki M. Bin Mahfoz. This is an open access article distributed under Creative Commons Attribution License [Attribution 4.0 International (CC BY 4.0)] 


\section{BACKGROUND}

Oral cancers are usually classified as head and neck cancers and include tumours of the oral cavity, oropharynx, nasopharynx, larynx, hypopharynx and lips. ${ }^{1}$ Oral cancer is a major health concern and the sixth leading cause of cancer related deaths worldwide. ${ }^{2}$ It also accounts for around $10 \%$ of all malignant neoplasms. ${ }^{3}$ Furthermore, oral cancers are a growing problem worldwide, with an expected incidence of about 448,000 cases and 228,000 deaths in $2018^{1}$ and about 389,000 new cases per annum. ${ }^{4}$ The incidence of oral cancer and its mortality rates are higher in underdeveloped countries than in the developed countries, ${ }^{5}$ of South-East Asia, where oral cancers account for up to $50 \%$ of all malignant tumours. ${ }^{5}$ Most studies have shown that treatment for oral cancer is most likely to be started at an advanced stage. ${ }^{3}$ In the Middle East, the prevalence of oral cancer has been reported to be $1.8-2.13$ / 100,000, ${ }^{6}$ and the incidence varies from 0.5 / 100,000 in Syria, ${ }^{7}$ to $10 / 100,000$ in the southern areas of Saudi Arabia, 8 with wide variation across the different regions in the country. A systematic review reported that the prevalence of oral cancers in Saudi Arabia ranged from $21.6 \%$ to $68.6 \%{ }^{9}$

There are many risk factors for oral cancer, including use of tobacco, excessive alcohol consumption, chewing betel quid, and various combinations of these habits. ${ }^{10}$ Other etiologic factors include ultraviolet exposure (lip cancer), chewing of areca nut, and human papillomavirus (HPV) infection. ${ }^{11}$ An infectious aetiology is associated with at least $20 \%$ of cancers worldwide, and viruses are responsible for around $15 \%$ of all human cancers. ${ }^{12}$ In one study, HPV was identified in up to 75 $\%$ of oropharyngeal tumour biopsies. ${ }^{11}$ In another study, approximately $4.5 \%$ of all oropharyngeal cancers were attributed to HPV, with an estimated 630,000 new cases diagnosed each year. ${ }^{13}$

HPV is a well-known sexually transmitted infection. Approximately 6 million persons are diagnosed to have HPV infection each year, and about $9.0 \%-13.0 \%$ of the world's population has already been infected. ${ }^{12}$ In a study performed in Saudi Arabia, HPV-DNA was detected in $9.8 \%$ of 417 women. ${ }^{14} \mathrm{HPV}$ has been associated with various types of human malignancies, including cervical, vulvar, vaginal, penile, anal and head and neck cancers. ${ }^{15}$

HPV related oropharyngeal cancers are closely linked to smoking and alcohol consumption. ${ }^{10}$ Some reports have shown that the risk of oral cancer is up to 15 times higher in smokers than in non-smokers. ${ }^{16}$ Although, consumption of alcohol is very low in Saudi Arabia, smoking is very common in men.

Furthermore, there is a large community of expatriates in Saudi Arabia, especially people from South-East Asia, where social chewing of areca nut in common. These factors are likely to result in an increased incidence of oral cancers in Saudi Arabia in the coming years. ${ }^{10}$

The high mortality rate associated with oral cancer can be explained by the late presentation in a large proportion of patients. ${ }^{17}$ Oral cancer has among the lowest 5-year survival rates of all cancers. ${ }^{18}$ Although the oral cavity is easily accessed for clinical examination, lack of awareness on the part of both patients and health care professionals leads to failure of early detection of precancerous and early cancerous oral lesions. ${ }^{19}$ The most effective way to improve the survival rate in patients with oral cancer is early detection, one study showed that patients with an early diagnosis of oral cancer had a higher survival rate that those who were diagnosed with the disease in its advanced stages. ${ }^{20}$ Therefore, increased awareness among the public and health care providers is key to improving the survival rate in patients with oral cancer.

The aim of this study was to assess the level of knowledge about oral cancer and its risk factors among dental and medical students at Majmaah University in Saudi Arabia. To our knowledge, this study is the first to address this topic and we anticipate that its findings will be useful at both the national and regional levels.

\section{METHODS}

This cross-sectional study was carried out on medical and dental students studying in Majmaah University from April 2020 to May 2020 after approval by the ethics committee at King Fahad Medical City. The study population was students studying in level 3 - 5 at university colleges of medicine and dentistry. Assuming the knowledge prevalence as of $50 \%$ and $\mathrm{q}(1-\mathrm{p})$ as $50 \%$ and confidence value of $95 \%$ and error of 5 $\%$, the study sample was calculated to be 385 based on the formula $\mathrm{N}=\mathrm{z}^{2} \mathrm{pq} / \mathrm{e}^{2}$. Prior consent was taken before administering the questionnaire. Questionnaire was distributed by simple random sampling among students. About 23 students didn't give consent for participation and 19 students filled the questionnaire incompletely, were excluded from the study making the final sample size of 343 . The questionnaire devised has section on information on demographic variables, including gender, age, academic level (undergraduate, intern, graduate, and postgraduate), and specialty. The other section of questionnaire contained 19 questions, each of which had five possible responses ("strongly agree", "somewhat agree", "somewhat disagree", "strongly disagree", and "I don't know").

\section{Statistical Analysis}

Data entry and analysis was done using SPSS version 23. Frequencies and percentages were calculated. Chi-square test was used to test the significant difference in proportions and percentages between the groups. $\mathrm{P}<0.05$ was considered to be statistically significant.

\section{RESULTS}

Among 343 students, 239 (70 \%) of respondents were males and $104(30.3 \%)$ were females. 160 (47\%) were dental students, and 183 (53\%) were medical students. About 180 $(52.5 \%)$ of respondents were in their fourth year of study, 157 $(45.8 \%)$ were in their fifth year, and $6(1.7 \%)$ were in their third year. The proportions of respondents in each year of study was distributed evenly between the medical and dental colleges (Table 1).

Most respondents, 288 (84\%) agreed strongly that smoking is a risk factor for oral cancer; about 205 (71\%) of these students were males, 152 (52.8\%) were medical students, and 148 (51.4\%) were in their fourth year of study. There was a significant relationship between awareness of 

dental), and year of study (all $\mathrm{P}<0.05$; Table 3 ).

About 238 (70\%) of respondents agreed strongly that chewing powdered tobacco products like gutka was also a risk factor for oral cancer; 162 (68\%) of these respondents were males, 124 (52\%) were dental students, and 126 (52.9\%) were in their fourth year. Overall, gender and specialisation were significantly associated with the respondents' awareness of chewing powdered tobacco products as a risk factor (both $\mathrm{P}$ $<0.05$; Table 3).

In contrast, only 84 (25\%) of respondents knew that HPV could cause oral cancer; 66 (78.60\%) were males, 58 (50\%) were medical students, and 43 (51.2\%) were in their fourth year. Gender, specialisation, and year of study were significantly associated with the respondents' knowledge about this risk factor (all $\mathrm{P}<0.05$; Table 3). Moreover, 56 (16.3 $\%)$ of respondents agreed strongly that chronic mechanical irritation was a risk factor for oral cancer; 32 (57.10\%) of these respondents were males, 23 (50\%) were medical students, and 38 (67.9\%) were in their fourth year. Gender, specialisation, and year of study were all significantly associated with the respondents' awareness of chronic mechanical irritation as a risk factor (Table 3).

Fifty-three percent of respondents had moderate knowledge about oral cancer, (12\%) had good knowledge, 29 $\%$ had poor knowledge, and $6 \%$ had no knowledge at all (Figure 1). There was no significant relationship between gender and knowledge about oral cancer; 272 (79.50\%) of the respondents with good knowledge were males, 102 (29.7\%) of those with moderate knowledge were females, 217 (63.4\%) of those with poor knowledge were males, and 82 (23.8\%) of those with no knowledge were females (Table 2). In contrast, there was a significant relationship between specialisation and had good knowledge were dental students, 181 (52.7\%) of those with moderate knowledge were medical students, 203 (59.4\%) of those with poor knowledge were dental students, and $203(71.4 \%)$ of those with no knowledge were medical students.

Year of study also had a significant effect on knowledge about oral cancer. In total, 167 (48.7\%) of students with good knowledge were in their fourth year, 158 (46.20\%) were in their fifth year; half of those with moderate knowledge were in their fourth year and $4(1.1 \%)$ were in their third year. Most $220(64.4 \%)$ of the students with poor knowledge were in their fourth year and 117 (33.7\%) in their fifth year. No student in their third year was aware of oral cancer whereas $262(76.2 \%)$ of those in their fifth year knew about the disease.

The level of respondents' knowledge about HPV as a risk factor for oral cancer is summarised in Table 4. We found that smoking as a risk factor and gender, specialisation (medical or knowledge about oral cancer; 70 (20.5\%) of respondents who

$58(16.9 \%)$ of respondents agreed strongly with the untrue statement that all types of HPV can cause oral cancer and 87 $(25.4 \%)$ somewhat agreed. We also found that 87 (25.4\%) of respondents agreed strongly that some types of HPV cause cervical cancer and $94(27.4 \%)$ somewhat agreed. In response to the untrue statement that HPV causes herpes and cold sores, 73 (20\%) of respondents strongly agreed, 96 (29\%) somewhat agreed, 44 (12\%) somewhat disagreed, 72 (20\%) strongly disagreed, and 62 (19\%) were unsure. When asked if HPV could cause acquired immune deficiency syndrome, 80 (23\%) strongly agreed, 54 (16\%) somewhat agreed, 70 (20 $\%$ ) somewhat disagreed, 76 (22\%) strongly disagreed, and 63 (18\%) were unsure. When asked if HPV is a sexually transmitted virus, most respondents agreed [155 (45\%) strongly, 92 (27 \%) somewhat], 35 (10 \%) strongly or somewhat disagreed, and 29 (9\%) were unsure. Most respondents agreed that HPV can be spread by deep kissing [163 (48\%) strongly, 94 (27\%) somewhat] and contaminated instruments [137 (40\%) strongly, 101 (30\%) somewhat]. The distribution of answers according to demographic characteristics is shown in Table 4.

Many respondents were confident that they had enough knowledge to diagnose oral cancer (20\% strongly agreed, 26 $\%$ somewhat agreed). Confidence was highest in male respondents, dental students, and those in their earlier years of study. However, most of the respondents reported that they had never encountered a case of oral cancer and did not know anybody with the disease (36.4\% strongly disagree, $39.7 \%$ I don't know). Table 5 shows the distribution of answers according to demographic characteristics.

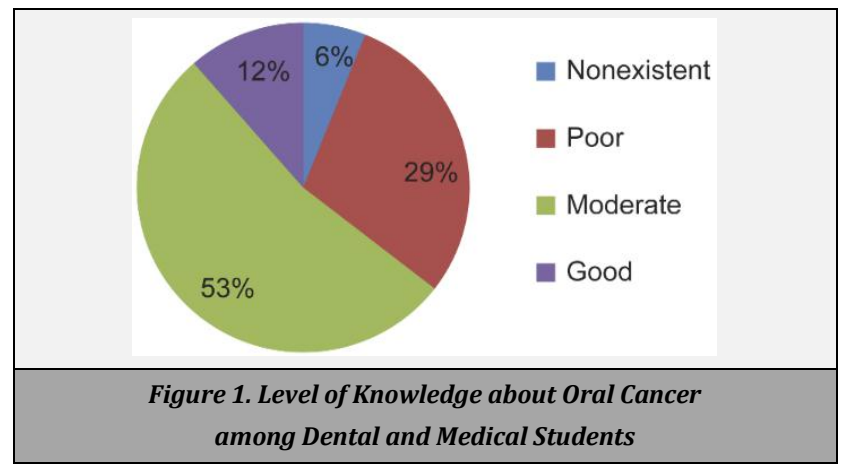

\begin{tabular}{|c|c|c|c|}
\hline \multirow{3}{*}{ Gender } & & $\mathbf{n}$ & $\%$ \\
\hline & Male & 239 & 69.7 \\
\hline & Female & 104 & 30.3 \\
\hline \multirow{2}{*}{ Specialization } & Dentistry & 160 & 46.6 \\
\hline & Medicine & 183 & 53.4 \\
\hline \multirow{4}{*}{ Year of study } & Third & 6 & 1.7 \\
\hline & Fourth & 180 & 52.5 \\
\hline & Fifth & 157 & 45.8 \\
\hline & Total & 343 & 100.0 \\
\hline \multicolumn{4}{|c|}{$\begin{array}{l}\text { Table 1. Demographic Distribution of Respondents } \\
\text { According to Specialization, Gender and Year of Study }\end{array}$} \\
\hline
\end{tabular}

\begin{tabular}{|c|c|c|c|c|c|c|}
\hline & & \multicolumn{4}{|c|}{ Level of Knowledge \% } & \multirow{2}{*}{ P-Value } \\
\hline & & Non-Existent & Poor & Moderate & Good & \\
\hline \multirow{3}{*}{ Gender } & Male & $261(76.2 \%)$ & $217(63.4 \%)$ & $241(70.3 \%)$ & $272(79.5 \%)$ & \multirow{3}{*}{0.247} \\
\hline & Female & $82(23.8 \%)$ & $126(36.6 \%)$ & $102(29.7 \%)$ & $71(20.5 \%)$ & \\
\hline & Total & $343(100 \%)$ & $343(100 \%)$ & $343(100 \%)$ & $343(100 \%)$ & \\
\hline \multirow{3}{*}{ Specialization } & Dental & $98(28.6 \%)$ & $203(59.4 \%)$ & $162(47.3 \%)$ & $70(20.5 \%)$ & \multirow{3}{*}{$0.001^{*}$} \\
\hline & Medical & $203(71.4 \%)$ & $140(40.6 \%)$ & $181(52.7 \%)$ & $273(79.5 \%)$ & \\
\hline & Total & $343(100 \%)$ & $343(100 \%)$ & $343(100 \%)$ & $343(100 \%)$ & \\
\hline \multirow{4}{*}{ Year of study } & Third & $0(0.0 \%)$ & $6(2.0 \%)$ & $4(1.1 \%)$ & $18(5.1 \%)$ & \multirow{4}{*}{$0.007^{*}$} \\
\hline & Fourth & $81(23.8 \%)$ & $220(64.4 \%)$ & $171(50.0 \%)$ & $167(48.7 \%)$ & \\
\hline & Fifth & $262(76.2 \%)$ & $117(33.7 \%)$ & $168(48.9 \%)$ & $158(46.2 \%)$ & \\
\hline & Total & $343(100 \%)$ & $343(100 \%)$ & $343(100 \%)$ & $343(100 \%)$ & \\
\hline \multicolumn{7}{|c|}{ Table 2. Respondents' Level of Knowledge According to Gender, Specialization and Year of Study } \\
\hline
\end{tabular}




\begin{tabular}{|c|c|c|c|c|c|c|c|c|c|}
\hline & & \multicolumn{2}{|c|}{ Gender \% } & \multicolumn{2}{|c|}{ Specialization \% } & \multicolumn{3}{|c|}{ Year of study \% } & \multirow{2}{*}{ Total \% } \\
\hline & & Male & Female & Dental & Medical & Third & Fourth & Fifth & \\
\hline \multirow{5}{*}{$\begin{array}{l}\text { I have sufficient } \\
\text { knowledge to diagnose } \\
\text { oral cancer }\end{array}$} & Strongly agree & 23.80 & 11.50 & 27.50 & 13.70 & 66.70 & 19.40 & 19.10 & 20.10 \\
\hline & Somewhat agree & 20.90 & 38.50 & 26.20 & 26.20 & 0.00 & 26.10 & 27.40 & 26.20 \\
\hline & Somewhat disagree & 23.80 & 22.10 & 18.80 & 27.30 & 33.30 & 21.70 & 24.80 & 23.30 \\
\hline & Strongly disagree & 20.50 & 7.70 & 10.00 & 22.40 & 0.00 & 20.00 & 13.40 & 16.60 \\
\hline & I don't know & 10.90 & 20.20 & 17.50 & 10.40 & 0.00 & 12.80 & 15.30 & 13.70 \\
\hline \multirow{5}{*}{$\begin{array}{l}\text { I have examined a case } \\
\text { of oral cancer or know a } \\
\text { person with oral cancer }\end{array}$} & Strongly agree & 10.50 & 13.50 & 15.00 & 8.20 & 0.00 & 13.90 & 8.90 & 11.40 \\
\hline & Somewhat agree & 3.30 & 5.80 & 6.20 & 2.20 & 0.00 & 2.20 & 6.40 & 4.10 \\
\hline & Somewhat disagree & 7.90 & 9.60 & 12.50 & 4.90 & 0.00 & 4.40 & 13.40 & 8.50 \\
\hline & Strongly disagree & 33.50 & 43.30 & 35.00 & 37.70 & 66.70 & 40.60 & 30.60 & 36.40 \\
\hline & I don't know & 44.80 & 27.90 & 31.20 & 47.00 & 33.30 & 38.90 & 40.8 & 39.70 \\
\hline
\end{tabular}

\section{DISCUSSION}

The aim of this questionnaire survey was to assess the level of knowledge of medical and dental students at Majmaah University about oral cancer and its risk factors. This research is important because HPV is likely to become the most common risk factor for oral cancer in the next decade. Furthermore, in Saudi Arabia, there are many risk factors for oral cancer that are more widespread than in other countries, such as smoking and chewing areca nut. Moreover, there is a relationship between HPV and cancerous oral lesions, ${ }^{21}$ which all doctors and dentists should be aware of to ensure that oral cancer can be diagnosed as early as possible

Overall, students in this study had moderate knowledge about oral cancer. However, only a quarter of students knew that HPV can cause oral cancer. Furthermore, only $45 \%$ of all questions related to HPV in this survey were answered correctly, which is consistent with the findings of other researchers. A study by Lewandowski et al. found that $43.4 \%$ of university students were unaware of any relationship between HPV and the risk of oral cancer. ${ }^{22}$

We found no significant relationship between student gender and level of knowledge, which can be explained by the educational system in Saudi Arabia being gender-neutral and the religion-based restrictions on extramarital sexual relationships in the country.

We anticipated that dental students would have a higher level of knowledge about oral cancer. However, we found that more questions were answered correctly by medical students than by dental students. A possible reason for this finding is that people tend to visit a doctor rather than a dentist when they have oral symptoms. ${ }^{23}$ For example, a study in the UK found that individuals considered to be at increased risk of oral cancer were more likely to consult their general practitioner than a dentist. ${ }^{24}$ Therefore, medical students may be are more aware of causes of oral pathology and the relationship between oral cancer and HPV. Moreover, most students in our study associated HPV more with other sexually transmitted diseases than with oral cancer.

We found that both medical students and dental students in their more advanced years of study had better knowledge about oral cancer and HPV, probably because they had received more training in oral pathology and were more likely to have read about or come into contact with a cases of oral cancer. This finding was in line with that of another study which reported that the mean oral cancer knowledge score increased with every year of academic study. ${ }^{25}$

Improving the awareness and knowledge of dental and medical students about oral cancer, including its relationship with HPV, is a key step towards increasing the likelihood of detection of the disease in its earlier stages and decreasing the associated high mortality and morbidity rates. Implementation of preventive measures, such as dental and medical counselling and lifestyle education programs, would help to reduce the morbidity and mortality rates that result from delayed detection. Medical and dental students' awareness and knowledge about oral cancer could be improved by revising the undergraduate curriculum to include more detailed information on risk factors, such as smoking and chewing areca nut, which are widespread in Saudi Arabia. One study in Saudi Arabia reported that $25 \%$ of the general adult population and $25 \%$ of university students were smokers; these rates are among the highest in the world. ${ }^{17}$ Saudi Arabia also has a large community of immigrants, particularly from South-East Asia, where areca nut chewing and smokeless tobacco consumption rates are high. ${ }^{17}$

This study has some limitations. This is a cross-sectional study conducted in one medical college; therefore findings may not generalize to medical and dental students at other institutions. Moreover, there was a low response rate from the third year students, compared to the response rate from students in the other levels. In general, this study had a relatively small sample size, again suggesting caution when generalizing the results. Given these limitations, further longitudinal studies with larger sample sizes must be carried out in medical colleges throughout Saudi Arabia to conclude more valid outcomes.

\section{CONCLUSIONS}

The findings of this study indicate that dental and medical students at Majmaah University have insufficient knowledge about oral cancer and its risk factors, particularly its relationship with HPV infection. This lack of knowledge is more apparent in dental students but had no association with gender. Improving the awareness and knowledge of dental and medical students about oral cancer, including its relationship with HPV, is a key step towards earlier detection of oral cancer and decreasing the mortality and morbidity rates associated with this disease. Preventive measures, such as dental and medical counselling and lifestyle programs, would help to decrease the high morbidity and mortality rates that result from delayed detection.

Data sharing statement provided by the authors is available with the full text of this article at jemds.com.

Financial or other competing interests: None. 
Disclosure forms provided by the authors are available with the full text of this article at jemds.com.

\section{REFERENCES}

[1] Bray F, Ferlay J, Soerjomataram I, et al. Global cancer statistics 2018: GLOBOCAN estimates of incidence and mortality worldwide for 36 cancers in 185 countries. CA Cancer J Clin 2018;68(6):394-424.

[2] Kamangar F, Dores GM, Anderson WF. Patterns of cancer incidence, mortality, and prevalence across five continents: defining priorities to reduce cancer disparities in different geographic regions of the world. J Clin Oncol 2006;24(14):2137-50.

[3] Siegel RL, Miller KD, Jemal A. Cancer Statistics, 2017. CA Cancer J Clin 2017;67(1):7-30.

[4] Kazmi F, Chaudhary MA, Mumtaz M, et al. Oral cancer knowledge and awareness amongst undergraduate dental students of Lahore - Pakistan. Pakistan Oral \& Dental Journal 2011;31(1):64-7.

[5] Krishna Rao SV, Mejia G, Roberts-Thomson K, et al. Epidemiology of oral cancer in Asia in the past decade - an update (2000-2012). Asian Pac J Cancer Prev 2013;14(10):5567-77.

[6] Al - Jaber A, Al - Nasser L, El - Metwally A. Epidemiology of oral cancer in Arab countries. Saudi Med J 2016;37(3):249-55.

[7] Shahrour MS, Badoura Z, Giko M. [Cancer of the jaw and oral cavity in the Syrian Arab Republic: an epidemiological study]. E Mediterr Health J 2005;11(3):273-86.

[8] Brown A, Ravichandran K, Warnakulasuriya S. The unequal burden related to the risk of oral cancer in the different regions of the Kingdom of Saudi Arabia. Community Dent Health 2006;23(2):101-6.

[9] Basha S, Mohamed RN, Al-Thomali Y, et al. The prevalence of oral cancer in Saudi Arabia: a systematic review. Ann Med Health Sci Res 2019;9:553-7.

[10] Lin WJ, Jiang RS, Wu SH, et al. Smoking, alcohol and betel quid and oral cancer: a prospective cohort study. J Oncol 2011;2011:525976.

[11] Argiris A, Karamouzis MV, Raben D, et al. Head and neck cancer. Lancet 2008;371(9625):1695-709.

[12] McLaughlin-Drubin ME, Munger K. Viruses associated with human cancer. Biochim Biophys Acta 2008;1782(3):127-50.

[13] Saraiya M, Unger ER, Thompson TD, et al. US assessment of HPV types in cancers: implications for current and 9 - valent HPV vaccines. J Natl Cancer Inst 2015;107(6):djv086.

[14] Suarez TP, Kelly JA, Pinkerton SD, et al. Influence of a partner's HIV serostatus, use of highly active antiretroviral therapy and viral load on perceptions of sexual risk behavior in a community sample of men who have sex with men. J Acquir Immune Defic Syndr 2001;28(5):471-7.

[15] Chaturvedi AK, Engels EA, Pfeiffer RM, et al. Human papillomavirus and rising oropharyngeal cancer incidence in the United States. J Clin Oncol 2011;29(32):4294-301.

[16] Lohavanichbutr P, Houck J, Fan W, et al. Genomewide gene expression profiles of HPV - positive and HPV negative oropharyngeal cancer: potential implications for treatment choices. Arch Otolaryngol Head Neck Surg 2009;135(2):180-8.

[17] Al - Haqwi AI, Tamim H, Asery A. Knowledge, attitude and practice of tobacco smoking by medical students in Riyadh, Saudi Arabia. Ann Thorac Med 2010;5(3):145-8.

[18] Warnakulasuriya S. Global epidemiology of oral and oropharyngeal cancer. Oral Oncol 2009;45(4-5):309-16.

[19] Lingen MW, Kalmar JR, Karrison T, et al. Critical evaluation of diagnostic aids for the detection of oral cancer. Oral Oncol 2008;44(1):10-22.

[20] Sargeran K, Murtomaa H, Safavi SM, et al. Survival after diagnosis of cancer of the oral cavity. Br J Oral Maxillofac Surg 2008;46(3):187-91.

[21] Candotto V, Lauritano D, Nardone M, et al. HPV infection in the oral cavity: epidemiology, clinical manifestations and relationship with oral cancer. Oral Implantol (Rome) 2017;10(3):209-20.

[22] Lewandowski B, Czenczek-Lewandowska E, Pakla P, et al. Awareness of Polish undergraduate and graduate students regarding the impact of viral infections and high - risk sexual behaviors on the occurrence of oral cancer. Medicine (Baltimore) 2018;97(41):e12846.

[23] Moreno-López LA, Esparza-Gómez GC, GonzálezNavarro A, et al. Risk of oral cancer associated with tobacco smoking, alcohol consumption, and oral hygiene: a case - control study in Madrid, Spain. Oral Oncol 2000;36(2):170-4.

[24] Carter LM, Ogden GR. Oral cancer awareness of general medical and general dental practitioners. Br Dent J 2007;203(5):E10.

[25] Hassona Y, Scully C, Tarboush AN, et al. Oral cancer knowledge and diagnostic ability among dental students. J Cancer Educ 2017;32(3):566-70. 